\section{Vagus nerve stimulation therapy (VNST) in epilepsy - implications for dental practice}

\author{
P. Lisowska ${ }^{1}$ and B. Daly ${ }^{2}$ \\ VERIFIABLE CPD PAPER
}

IN BRIEF

- Highlights the principles of epilepsy control.

- Raises awareness that patients with intractable epilepsy may use VNST to control epileptic seizures.

- Demonstrates how VNST is used in the management of intractable epilepsy and how VNST operates.

- Discusses the implications of VNST use including medical devices interactions and safety features affecting dental treatment.

Epilepsy is a chronic condition which affects about 1\% of the population. It is important that the dental team is aware of the management of epileptic seizures and epileptic syndromes including recent advances in seizure management. As people with epilepsy often get a warning aura before seizures begin, the management of the condition has increasingly involved measures to prevent the seizure, once the aura has begun. Vagus nerve stimulation therapy (VNST) in epilepsy involves the use of an implantable electronic device and is being increasingly used in the UK to control severe treatment resistant epilepsy. As a result, more patients will be presented to clinicians in the primary healthcare setting and hospital services with these devices in place. Members of the dental team need to understand the principles of epilepsy control, how VNST is used in the management of intractable epilepsy, how the VNST system operates and the implications of VNST use for dental practice including medical devices, interactions and safety features.

\section{INTRODUCTION}

The worldwide prevalence of epilepsy is estimated at 0.5 to $0.9 \%$ in the general population, affecting 50 million people. ${ }^{1}$ The societal costs of epilepsy in Europe are high with the cost of epilepsy estimated at 15.5 billion Euros in 2004, with 55\% covering indirect costs, namely sick leave and premature mortality, and 45\% encompassing medical and social care. ${ }^{1} \mathrm{An}$ epileptic seizure is defined as an episodic disturbance of movement, feeling or consciousness that can be caused by sudden synchronous, inappropriate and excessive electrical discharges that interfere with the normal function of the brain. ${ }^{2}$ The associated loss of consciousness may result in generalised injuries and dental trauma. In the Textbook and colour atlas of traumatic injuries to teeth, a study citing 437 patients with epilepsy showed that 52\% had suffered traumatic dental injuries, one third of

"King's College London Dental Institute, Department of Community Special Care Dentistry, King's College London, Denmark Hill Campus, Bessemer Road, SE5 9RW; ${ }^{2}$ King's College London Dental Institute, Unit of Academic Special Care Dentistry, King's College London, Denmark Hill Campus, Bessemer Road, SE5 9RW

${ }^{*}$ Correspondence to: Phidelma Lisowska

Email: phidelma.lisowska2@nhs.net;

Tel: 02032993480

\section{Refereed Paper}

Accepted 3 November 2011

DOI: 10.1038/sj.bdj.2012.47

${ }^{\circledR}$ British Dental Journal 2012; 212: 69-72 these being directly related to falls during epileptic seizures. ${ }^{3}$ Seizure control is vitally important in reducing this burden and improving the quality of life for the individuals concerned. As people with epilepsy often get a warning aura before seizures begin, the management of the condition has increasingly involved measures to prevent and reduce the frequency or duration of the seizures once the aura has begun. One such therapy is vagus nerve stimulation therapy $\left(\right.$ VNST) ${ }^{4}$ which is an implantable electrical device providing brain stimulation in order to reduce the frequency and duration of epileptic seizures and is particularly used in people who have refractory epilepsy where surgical management is not advisable. ${ }^{5,6}$ As VNST is now becoming more widely used in the management of epilepsy, the dental team is likely to encounter patients using VNST in primary healthcare, as well as in a hospital setting. It is important therefore, that they understand the function, risks and benefits of VNST and the implications for dental care planning and safety issues associated with the device.

\section{EPILEPSY}

Epilepsy has been described as 'a chronic disease characterised by the risk of recurrent seizures.' Epilepsy is classified on a multi-axial basis with axes that include the epileptic seizure, the epileptic syndrome and the aetiology. ${ }^{8,9}$ Management of the condition in the first instance is normally through medication with about two thirds of people having their condition adequately controlled in this way. ${ }^{5}$ In some patients prescribed with dialantin, high plaque and calculus deposits are associated with gingival hyperplasia in long term users. ${ }^{10}$ In all cases of epilepsy drug combinations need to be carefully monitored to assess drug interactions and side effects such as drowsiness, mental and physical coordination problems and blood dyscrasias. About 30\% of people are said to have treatment resistant epilepsy (TRE) or have unacceptable side effects from treatment., ${ }^{5,6}$ For TRE which is not amenable to drug control some surgical interventions may be required, the most common being temporal lobectomy. ${ }^{5-11}$ The less invasive and minor procedure of vagus nerve stimulation therapy (VNST) is now gaining acceptance., ${ }^{5,611}$ Patients who previously would have required specialist dental care due to the severity of their epilepsy, may now be treated in the primary dental care setting with these devices in place.

\section{VAGUS NERVE STIMULATION THERAPY (VNST)}

The use of VNST is a well established method of epilepsy control which was first introduced in 1997.,5,11 VNST is usually introduced for management of 
severe epilepsy in adults and adolescents over twelve years of age, when seizures are uncontrolled by anti-epileptic drugs (AEDs) or the side effects of drugs are unacceptable. ${ }^{5}$ VNST controls severe epilepsy by acting synergistically with AEDs. ${ }^{5}$

The VNST system consists of a pulse generator implanted below the left collar bone in a surgically made pocket of skin, with the electrode implanted in the left vagus nerve in the patient ${ }^{4}$ (Fig. 1). The VNST system sends a mild electrical stimulation to the brain, via the vagal A fibres (the largest and most myelinated) of the left vagus nerve, affecting the reticular activating system and limbic systems of the brain. ${ }^{12}$ The mechanisms of action of VNST have been investigated using anatomicphysiologic studies, experimental epilepsy studies and human imaging, EEG and CSF studies. ${ }^{13}$ It is postulated that anti-seizure mechanisms are produced by altered vagal afferent activities affecting the reticular activating system, the central autonomic network, the limbic system and the diffuse noradrenergic projection system. ${ }^{13}$ Altered blood flow and synaptic neuro-transmitters appear to play a key role. ${ }^{13}$

The external system to calibrate the VNS pulse generator consists of a programming wand attached to computer software. When the VNS pulse generator is inserted under the left collar bone by the neurosurgeon, the neurosurgeon sets the pulse generator to 'normal mode' using a computer and wand system at the hospital. This calibrates and fine tunes the VNS pulse generator to fire off regularly, producing a base-line pulse, to prevent epileptic seizures. When the patient starts to have an aura (a warning sign which may be a change in auditory perception, vision or olfactory sensation), the patient uses the cyberonics magnet (which can look like a watch or pager). The magnet is slowly swiped across the vagus nerve stimulator to activate the device's reed switch, which stimulates the vagus nerve and brain, to curtail the epileptic seizure. A member of the patient's family or a carer can also assist the patient by swiping the magnet across the vagus nerve therapy device under the collar bone. In the event that the epileptic seizure does not abate, some patients are advised by their GMPs and neurologists to carry midazolam for buccal use to supplement use of the vagus

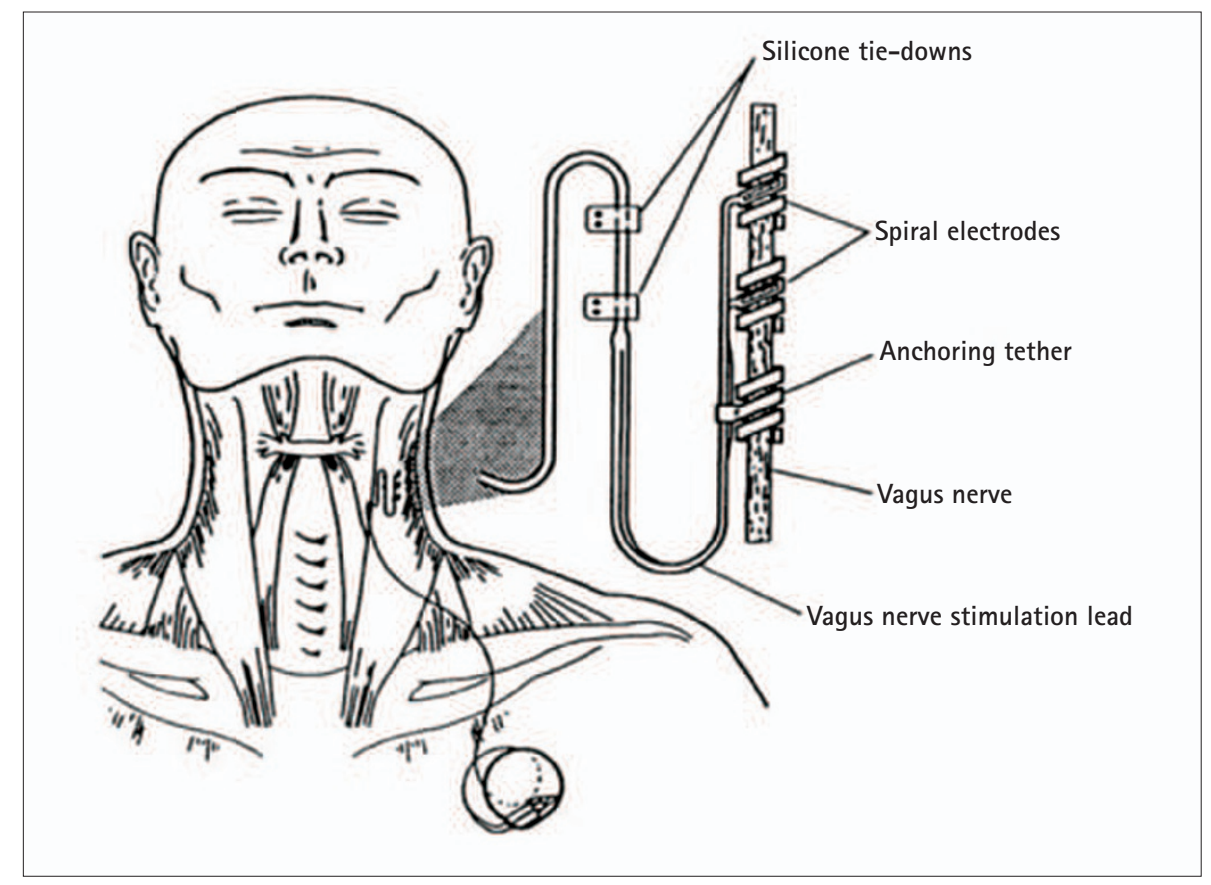

Fig. 1 Implanted programmable stimulator in the chest with detail of stimulating electrode. Cyberonic Inc - with permission from Wolters Kluwer

nerve stimulator. The effectiveness of the use of buccal midazolam is the subject of a consultation on a NICE guideline draft. ${ }^{14}$

Randomised controlled trials undertaken before the introduction of VNST in the USA in 1997 and subsequent nonrandomised controlled trials undertaken in children and adults after 1997 suggest that VNST results in improved seizure control, reduced seizure frequency and reduced seizure duration. ${ }^{5,6,11}$ Other potential benefits included decreased dosage of AEDs and reduced hospitalisation. ${ }^{5}$ Current evidence suggests that VNST is efficacious, effective and safe. ${ }^{5,6,11}$

\section{COMMON ADVERSE EFFECTS OF VAGUS NERVE STIMULATION THERAPY}

VNST devices cause adverse effects pertaining to chronic vagus nerve stimulation. The common respiratory side effects of chronic vagus nerve stimulation include dyspnoea on exertion, altered breathing patterns, snoring during sleep and obstructive sleep apnoea. ${ }^{4,15}$ Stimulation of the vagus nerve may lead to swallowing difficulties and aspiration of fluid into the lungs. The vagus nerve may become permanently damaged due to, for example, over stimulation through frequent use of the magnet. Reversible altered vocalisation and somatic sensation effects may occur, which quickly reverse once stimulation ceases. ${ }^{4}$

\section{CLINICAL IMPLICATIONS}

\section{Primary dental care settings}

Many patients who have previously had uncontrolled epilepsy may now attend primary dental care. Dentists and the dental team need to be aware of the VNST device and the use of the cyberonics magnet. The routine management of epilepsy involves clarification of the diagnosis and type of epileptic seizures, any known stimuli, warning signs and appropriate management should seizures occur. All patients with epilepsy should be asked if they are using a VNST device and full details of the device and how it is operated should be recorded in their notes. Devices typically are either in a watch-style format or a pager-style clipped to a waist belt. Details should be provided of any aura or warning signs and the agreed signal for the patient to indicate the onset of an aura or seizure. Patients should be reminded to bring any fit charts if the epilepsy is unstable. Regular medication must be taken to prevent epileptic seizures and the cyberonic magnet and buccal midazolam (if recommended by GMP) made available to the dental team at each appointment. It is important that the patient's condition is reviewed at each dental visit. Routine dental care should not be problematic, provided high speed suction or rubber dam is used. In order to minimise seizure onset in 
the dental surgery, it is suggested that the dental team ensures that the patient has taken their AEDs and are not dehydrated, stressed, anxious or in pain. Incipient infection should be rapidly addressed.

\section{The effect of VNST devices on pain management and altered taste and smell}

Low intensity VNST leads to a reduction in thermal pain thresholds, as measured by quantitative sensory testing. ${ }^{16}$ The dental team need to be aware that people using the VNST device may have reduced pain thresholds. Trigeminal pain may also occur with VNST as a side effect resulting in unnecessary dental investigation and treatment. ${ }^{17}$ The trigeminal pain is reversible with a decrease in stimulation current intensity. In addition altered taste and smell perception have been reported. ${ }^{18}$

\section{The effect of electrical dental equipment on VNST device function}

The effect of electrical dental equipment including Cavitron ultra-sonic scalers, Automix amalgamators, Analytic pulp testers and Optilux high output curing lights on VNST device function were evaluated by Roberts. ${ }^{19}$ None of the dental devices generated a magnetic field of sufficient magnitude to affect the pulse generator's normal function. However, the use of diathermy and cardiac defibrillators are contra-indicated in any patient with a vagus nerve stimulator. ${ }^{4}$ External cardiac defibrillators are known to deliver large amounts of energy and can damage the pulse generator. The paddles need to be placed as far away from the pulse generator as possible, perpendicular to the pulse generator and the lowest clinically appropriate output setting possible. During a medical emergency involving a cardiac arrest, the cardiac defibrillator paddles must not be placed over the pulse generator on the left side. A small surgical scar under the left collar bone will give an indication of the area to avoid during paddle placement. The pulse generator must be checked for correct function after defibrillation. ${ }^{20}$

No short wave diathermy, microwave diathermy or therapeutic ultra-sound diathermy should be used during dental treatment. Energy from diathermy heats the
VNST system causing vagus nerve damage, vascular or tissue damage. If there is damage to the blood vessels in the neck this may result in pain and loss of vocal cord function or even death. Diagnostic ultra-sound is not included as a contraindication by the manufacturer. ${ }^{4}$

It is worth pointing out that the pulse generator of the VNST device may interfere with hearing aids operating in the $30 \mathrm{kHz}$ to $100 \mathrm{kHz}$ range. ${ }^{4}$ This needs to be considered for hearing impaired patients, carers or healthcare professionals providing care for people with epilepsy.

\section{Anaesthetic considerations during dental treatment under general anaesthesia}

During the pre-operative assessment it is important to note in the dental record the date of the VNST implantation and the most recent check. It is also important to record whether any anaesthetic complications during insertion have occurred, the severity of symptoms when the device is turned off and the current medications taken by the patient and reported adherence with the medication regime.

The surgical team who implanted the VNST device should be made aware of the approaching operation and should liaise with the anaesthetist and dental team to discuss safety, equipment conflicts, programming the device, medication changes when the VNST device is switched off and postoperative evaluation of the VNST device.

It is important that the anaesthetist and dental team are aware that there may be laryngeo-pharyngeal dysfunction with increased risk of aspiration of gastric contents. ${ }^{15}$ Abnormal motion of the vocal chords can cause partial obstruction with a laryngeal mask airway, so the anaesthetic team may want to consider alternative airway management. ${ }^{21}$

Other considerations include maintaining the humidity and temperature of the operating theatre within the recommended ranges (that is, temperature at 20 to $24^{\circ} \mathrm{C}$ and humidity at between 50 to $60 \%$ ) to reduce the risk of producing static electricity. ${ }^{22}$

Postoperatively, discharge procedures would routinely include a check of the VNST device for any damage or malfunction.

\section{The effect of use of specialist diagnostic equipment on VNST device function}

Patients can suffer serious injuries or death due to device interactions as outlined previously. Devices may also include peripheral nerve stimulation and magnetic resonance imaging. ${ }^{19}$ Radiologists providing imaging for tumours, trauma and wisdom teeth assessments should seek advice on the use of MRI scans from the manufacturers as there is a risk of the electrodes attached to the VNS overheating with the magnetic resonance body coil in the transmit mode.

Oncology teams dealing with head and neck cancer will be aware that radiation, cobalt machines and linear accelerators may damage the pulse generator and there is a need to seek guidance from the manufacturer on possible interactions.

\section{OTHER IMPLANTABLE ELECTRICAL DEVICES}

There are currently many clinical applications in electrotherapy and neuromodulation for implantable electrical devices. These devices provide an alternative treatment option, where medication alone cannot control disease symptoms. Deep brain stimulators control Parkinson's disease, obsessive-compulsive disorder and chronic pain. Sacral nerve stimulators are indicated for incontinence and pelvic pain. Phrenic nerve stimulators form a diaphragmatic pacemaker to control breathing in cervical spinal cord injuries. Patients in the UK may also be present in the primary care setting with vagus nerve stimulation for treatment-resistant depression. However NICE (2009) has not given full approval for this procedure, as there is not much good evidence about safety or how well this procedure works. ${ }^{21}$

Other devices include spinal cord stimulators, gastric pacemakers and bone stimulators. Increasingly, laryngeal pacemakers are being fitted to prevent aspiration in stroke related dysphasia. Many of the principles for the clinical management of vagus nerve stimulators also apply to other implantable electrical devices.

\section{CONCLUSIONS}

VNST is now becoming a more common method of managing treatment resistant epilepsy. Many clinicians may be seeing 
people in primary care who previously have had to have specialist care because of their uncontrolled seizures. The dental team need to be aware of current approaches in prevention and management of epileptic seizures including the VNST device. Taking a good history, maintaining accurate records, deciding a clear agreement on aura signals and the roles and responsibilities of the dental team during seizure onset are essential parts of patient management. Collaboration with the patient's GMP and medical team is essential to ensure that any dental procedure does not damage or interfere with VNST. It should be noted that patients may be wearing implantable devices for other therapeutic reasons and so a careful medical history is always integral.

The authors wish to thank the late Dr Liana Zoitopoulos (Head of the Department of Community Special Care Dentistry, Consultant in Special Care Dentistry and Honorary Senior Lecturer) for setting up the Research Steering Group in the Department.

1. Pugliatti $M$, Beghi E, Forsgren $L$, Ekman $M$, Sobocki P. Estimating the cost of epilepsy in Europe: a review with economic modelling. Epilepsia 2007;
48: 2224-2233.

2. Brodie M J, French J A. Management of epilepsy in adolescents and adults. Lancet 2000; 356: 323-329.

3. Andreasen J 0 , Andreasen F M, Anderson L. Textbook and colour atlas of traumatic injuries to teeth. London: Wiley-Blackwell, 2007.

4. Cyberonics. Epilepsy patient's manual for vagus nerve stimulation with the VNS Therapy ${ }^{T M}$ system. Houston: Cyberonics Inc., 2008.

5. Milby $\mathrm{A} H$, Halpern $\mathrm{C} H$, Baltuch $\mathrm{G} H$. Vagus nerve stimulation in the treatment of refractory epilepsy. Neurotherapeutics 2009; 6: 228-237.

6. Kwan P Brodie M J. Early identification of refractory epilepsy. N Eng/ J Med 2000; 342: 314-319.

7. Aragon CE, Burneo J C. Understanding the patient with epilepsy and seizures in dental practice. J Can Dent Assoc 2007; 73: 71-76.

8. Berg A T, Scheffer I E. New concepts in classification of the epilepsies: entering the 21st century. Epilepsia 2011; 52: 1058-1062.

9. National Institute for Health and Clinical Excellence. The epilepsies: the diagnosis and management of the epilepsies in adults and children in primary and secondary care (partial update of NICE clinical guideline 20). NICE guideline. Draft for consultation. London: NICE, 2011. Online article available at http://www.nice.org.uk/nicemedia/ live/12108/52447/52447.pdf (accessed 27/10/2011).

10. Lucchesi J A, Cortelli S C, Rodrigues J A, Duarte P M. Severe phenytoin-induced gingival enlargement associated with periodontitis. Gen Dent 2008; 56: 199-203.

11. Elliott R E, Morsi A, Kalhorn S et al. Vagus nerve stimulation in 436 consecutive patients with treatment-resistant epilepsy: long-term outcomes and predictors of response. Epilepsy Behav 2011; 20: $57-63$.

12. Woodbury D M, Woodbury J W. Effects of vagal stimulation on experimentally induced seizures in rats. Epilepsia 1990; 31(Suppl 2): s7-s19.

13. Henry T R. Therapeutic mechanisms of vagus nerve stimulation. Neurology 2002; 59: s3-s14.

14. National Institute for Health and Clinical Excellence: Vagus nerve stimulation for treatment-resistant depression. CG330. London: NICE, 2009. Online article available at http://www.nice.org.uk/nicemedia/ live/12149/46667/46667.pdf (accessed 27/10/11).

15. Banzett R B, Guz A, Paydarfar D, Shea S A Schachter S C, Lansig R W. Cardiorespiratory variables and sensation during stimulation of the left vagus nerve in patients with epilepsy. Epilepsy Res 1999; 35: 1-11.

16. Ness T J, Fillingim R B, Randich $A$, Backensto E M Faught $E$. Low intensity vagal nerve stimulation lowers human thermal pain thresholds: Pain 2000; 86: 81-85.

17. Carius A, Schulze-Bonhage A. Trigeminal pain under vagus nerve stimulation. Pain 2005; 118: 271-273.

18. Kirchner A, Landis B N, Haslbeck M, Stefan $H_{1}$ Renner B, Hummel T. Chemosensory function in patients with vagal nerve stimulators. J Clin Neurophysio/ 2004; 21: 418-425.

19. Roberts H W. The effect of electrical dental equipment on a vagus nerve stimulator's function. $J A m$ Dent Assoc 2002; 133: 1657-1664.

20. Venkatraghavan L, Chinnapa V, Peng P, Brull R. Non-cardiac implantable electrical devices: brief review and implications for anaesthesiologists. Can J Anaesth 2009; 56: 320-326.

21. Bernards C M. An unusual cause of airway obstruction during general anaesthesia with a laryngeal mask airway. Anesthesiology 2004; 100: 1017-1018.

22. Association of periOperative Registered Nurses. AORN guidance statement: care of the perioperative patient with an implanted electronic device. AORN J 2005; 82: 74-82, 85-90, 93-98 passim. 